\title{
Erratum to: Aurantibacter crassamenti gen. nov., sp. nov., a bacterium isolated from marine sediment
}

\author{
Jaewoo Yoon ${ }^{1} \cdot$ Hiroaki Kasai $^{2}$
}

Published online: 24 October 2016

C) Springer-Verlag Berlin Heidelberg 2016

\section{Erratum to: Archives of Microbiology DOI:10.1007/s00203-016-1280-y}

Subsequent to the publication of the above paper, it has been noticed that the characteristics of strain Kriegella aquimaris $\mathrm{KMM} 3665^{\mathrm{T}}$ in Table 1, cellular fatty acids for strain Kriegella aquimaris KMM $3665^{\mathrm{T}}$ in Table 2 and GenBank/EMBL/DDBJ accession number of strain Aurantibacter crassamenti $\mathrm{HG} 732^{\mathrm{T}}$ in Fig. 2 were incorrectly published. The corrected Tables 1 and 2 and Fig. 2 are provided here.

The online version of the original article can be found under doi:10.1007/s00203-016-1280-y.

Jaewoo Yoon

jwyoon@kmu.ac.kr

1 College of Pharmacy, Keimyung University, 1095

Dalgubeoldaero, Dalseo-Gu, Daegu 42601,

Republic of Korea

2 Marine Biosciences Kamaishi Research Laboratory,

Kitasato University, 160-4, Utou, Okirai, Sanriku-cho,

Ofunato, Iwate 022-0101, Japan 
Table 1 Differential characteristics of strain $\mathrm{HG}_{732^{\mathrm{T}}}$ and other closely related taxa

\begin{tabular}{|c|c|c|c|c|c|}
\hline Characteristic & 1 & 2 & 3 & 4 & 5 \\
\hline Pigmentation & Orange & Orange to dark orange & $\begin{array}{l}\text { Yellow to dark red } \\
\left.\text { (yellow }{ }^{b}\right)\end{array}$ & $\begin{array}{l}\text { Yellow to dark orange } \\
\quad\left(\text { yellow }^{\text {d }}\right)\end{array}$ & $\begin{array}{l}\text { Orange to dark orange } \\
\left(\text { dark orange } \mathrm{e}^{\mathrm{e}}\right)\end{array}$ \\
\hline Gliding motility & - & $+^{\mathrm{a}}$ & + & $\pm\left(+^{\mathrm{d}}\right)$ & $\pm\left(-{ }^{e}\right)$ \\
\hline $\begin{array}{l}\text { Temperature range for } \\
\text { growth }\left({ }^{\circ} \mathrm{C}\right)\end{array}$ & $20-37$ & $4-37^{\mathrm{a}}$ & $13-45$ & $4-50\left(4-33^{\mathrm{d}}\right)$ & $4-40\left(10-37^{\mathrm{e}}\right)$ \\
\hline $\mathrm{pH}$ range for growth & $6-9$ & $6-8$ & $6-8.5$ & $5.5-10.5\left(5.5-10^{\mathrm{d}}\right)$ & $6-10$ \\
\hline $\begin{array}{l}\text { Highest } \mathrm{NaCl} \text { tolerance } \\
(\%, \mathrm{w} / \mathrm{v})\end{array}$ & 5 & $6^{\mathrm{a}}$ & 10 & 10 & $8\left(7^{\mathrm{e}}\right)$ \\
\hline Nitrate reduction & - & - & + & $\pm\left(+^{\mathrm{d}}\right)$ & $\pm\left(+^{e}\right)$ \\
\hline \multicolumn{6}{|l|}{ Hydrolysis of } \\
\hline Gelatin & - & + & + & $\pm\left(-{ }^{\mathrm{d}}\right)$ & $\pm-\left(-^{\mathrm{e}}\right)$ \\
\hline ONPG & + & - & + & $\pm\left(-{ }^{\mathrm{d}}\right)$ & + \\
\hline Starch & - & - & $\pm\left(+^{\mathrm{b}}\right)$ & $\pm\left(+^{\mathrm{d}}\right)$ & - \\
\hline Urea & - & + & - & - & $\pm\left(+^{\mathrm{e}}\right)$ \\
\hline \multicolumn{6}{|l|}{ Acid production from } \\
\hline Cellobiose & - & - & $\pm\left(+{ }^{c}\right)$ & $\pm\left(-{ }^{\mathrm{d}}\right)$ & + \\
\hline Galactose & - & - & - & $\pm\left(-^{\mathrm{d}}\right)$ & $\pm\left(+^{\mathrm{e}}\right)$ \\
\hline Glucose & - & - & $\pm\left(-{ }^{c}\right)$ & $\pm\left(-{ }^{\mathrm{d}}\right)$ & + \\
\hline Maltose & - & - & $\pm\left(+{ }^{c}\right)$ & $\pm\left(-{ }^{\mathrm{d}}\right)$ & $\pm\left(+^{\mathrm{e}}\right)$ \\
\hline Melibiose & - & - & - & $\pm\left(-{ }^{\mathrm{d}}\right)$ & $\pm\left(-{ }^{\mathrm{e}}\right)$ \\
\hline Lactose & - & - & - & $\pm\left(-{ }^{\mathrm{d}}\right)$ & + \\
\hline Raffinose & + & - & $\pm\left(-{ }^{c}\right)$ & $\pm\left(-{ }^{\mathrm{d}}\right)$ & $\pm\left(+^{\mathrm{e}}\right)$ \\
\hline Polar lipids & PG, 3UAL, 2UL & ND & ND & PE, 2UAL, 4UL & PE, 2UL \\
\hline $\begin{array}{l}\text { DNA G }+\mathrm{C} \text { content } \\
(\mathrm{mol} \%)\end{array}$ & 35.2 & $39.2^{\mathrm{a}}$ & $36.1-43.4\left(43^{\mathrm{c}}\right)$ & $34.2-41.1\left(37.5^{\mathrm{d}}\right)$ & $37-40\left(37.5-38.2^{\mathrm{e}}\right)$ \\
\hline
\end{tabular}

Strains: $1 \mathrm{HG} 732^{\mathrm{T}}$ (Aurantibacter crassamenti gen. nov., sp. nov.; present study), 2 Kriegella aquimaris KMM $3665^{\mathrm{T}}$ (data from this study), 3 Zobellia spp. (Barbeyron et al. 2001; Nedashkovskaya et al. 2004b), 4 Maribacter spp. (Nedashkovskaya et al. 2004a; Zhang et al. 2009; Hu et al. 2015; Jackson et al. 2015; Park et al. 2015), 5 Arenibacter spp. (Ivanova et al. 2001; Nedashkovskaya et al. 2006; Sun et al. 2010; Jeong et al. 2013)

Symbols: $P E$ phosphatidylethanolamine, $P G$ phosphatidylglycerol, $U A L$ unidentified aminolipid, $U L$ unidentified lipid, + positive, - negative, $N D$ no data

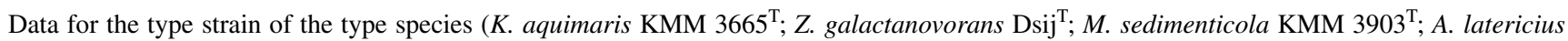
KMM 426 ${ }^{\mathrm{T}}$ ) from a, Nedashkovskaya et al. 2008; b, Barbeyron et al. 2001; c, Nedashkovskaya et al. 2004b; d, Nedashkovskaya et al. 2004a; e, Ivanova et al. 2001 
Table 2 Comparison of cellular fatty acids for strain $\mathrm{HG} 732^{\mathrm{T}}$ and other closely related taxa

\begin{tabular}{llllll}
\hline Fatty acid & 1 & 2 & 3 & 4 & 5 \\
\hline iso-C13:0 & $\operatorname{tr}$ & $\operatorname{tr}$ & - & $\operatorname{tr}$ & $\operatorname{tr}-1.1$ \\
C13:0 & - & - & - & - & - \\
iso-C14:0 & - & - & - & 1.4 & - \\
C14:0 & 1.4 & $\operatorname{tr}$ & $\operatorname{tr}-1.0$ & $\operatorname{tr}-1.7$ & $\operatorname{tr}-1.0$ \\
C15:0 & - & 11.1 & $7.5-14.4$ & $2.9-15.5$ & $4.1-22$ \\
C15:0 2-OH & $\operatorname{tr}$ & - & - & $\operatorname{tr}$ & $\operatorname{tr}-1.0$ \\
C15:0 3-OH & - & - & $\operatorname{tr}$ & $1.4-3.9$ & $\operatorname{tr}-2.2$ \\
iso-C15:0 & 33.1 & 12.3 & $16.8-22.5$ & $9.4-42.6$ & $6.8-15$ \\
iso-C15:0 3-OH & 4.3 & 3.2 & $4.6-8.3$ & $2.0-12.6$ & $3.5-7.2$ \\
iso-C15:1 G & 10.5 & 19.7 & - & $5.2-16.7$ & $9.3-12.7$ \\
anteiso-C15:0 & 1.2 & $\operatorname{tr}$ & $1.0-1.8$ & $\operatorname{tr}-9.0$ & $1.8-13.5$ \\
C15:1 $\omega 6 c$ & 2.6 & 2.5 & $1.1-3.2$ & $\operatorname{tr}-4.8$ & $\operatorname{tr}-4.2$ \\
C16:0 & 1.3 & $\operatorname{tr}$ & $\operatorname{tr}-2.6$ & $\operatorname{tr}-1.9$ & $\operatorname{tr}-2.7$ \\
C16:0 3-OH & 1.4 & $\operatorname{tr}$ & $2.4-4.9$ & $1.1-5.5$ & $\operatorname{tr}-3.7$ \\
iso-C16:0 & - & $\operatorname{tr}$ & - & $\operatorname{tr}-1.8$ & $\operatorname{tr}-1.7$ \\
iso-C16:0 3-OH & $\operatorname{tr}$ & $\operatorname{tr}$ & - & $\operatorname{tr}-6.8$ & $\operatorname{tr}-7.2$ \\
C17:0 2-OH & 1 & $\operatorname{tr}$ & - & $1-3.8$ & $\operatorname{tr}-5.1$ \\
C17:1 $\omega 6 c$ & $\operatorname{tr}$ & $\operatorname{tr}$ & $1.0-1.2$ & $\operatorname{tr}-1.7$ & $\operatorname{tr}-3.0$ \\
iso-C17:1 $\omega 9 c$ & - & 6.4 & $2.4-5.1$ & $2.0-9.4$ & $2.2-5.3$ \\
iso-C17:0 3-OH & 22.2 & 12.8 & $15.1-25.9$ & $4.8-29.2$ & $6.9-21.9$ \\
C18:0 & - & $\operatorname{tr}$ & - & $\operatorname{tr}$ & - \\
Summed feature 3 & 8.2 & 9.4 & $9.9-15.5$ & $1.7-14.8$ & $9.6-20.4$ \\
Summed feature $9^{\mathrm{b}}$ & 6.2 & - & - & 7.9 & - \\
\hline & & & & & \\
\hline
\end{tabular}

Strains: 1 HG732 ${ }^{\mathrm{T}}$ (Aurantibacter crassamenti gen. nov., sp. nov.; present study), 2 Kriegella aquimaris KMM $3665^{\mathrm{T}}$ (Nedashkovskaya et al. 2008), 3 Zobellia spp. (Barbeyron et al. 2001; Nedashkovskaya et al. 2004b), 4 Maribacter spp. (Nedashkovskaya et al. 2004a; Zhang et al. 2009; Hu et al. 2015; Jackson et al. 2015; Park et al. 2015), 5 Arenibacter spp. (Ivanova et al. 2001; Nedashkovskaya et al. 2006; Sun et al. 2010; Jeong et al. 2013)

Symbols: $\operatorname{tr}$ trace (less than $1.0 \%$ ), - not detected. The data were typically obtained by GLC using the MIDI system

${ }^{a}$ Summed feature 3 consists of $\mathrm{C} 16: 1 \omega 7 c$ and/or $\mathrm{C} 16: 1 \omega 6 c$

b Summed feature 9 consists of C16:0 10-methyl/iso-C17:1 $\omega 9 c$

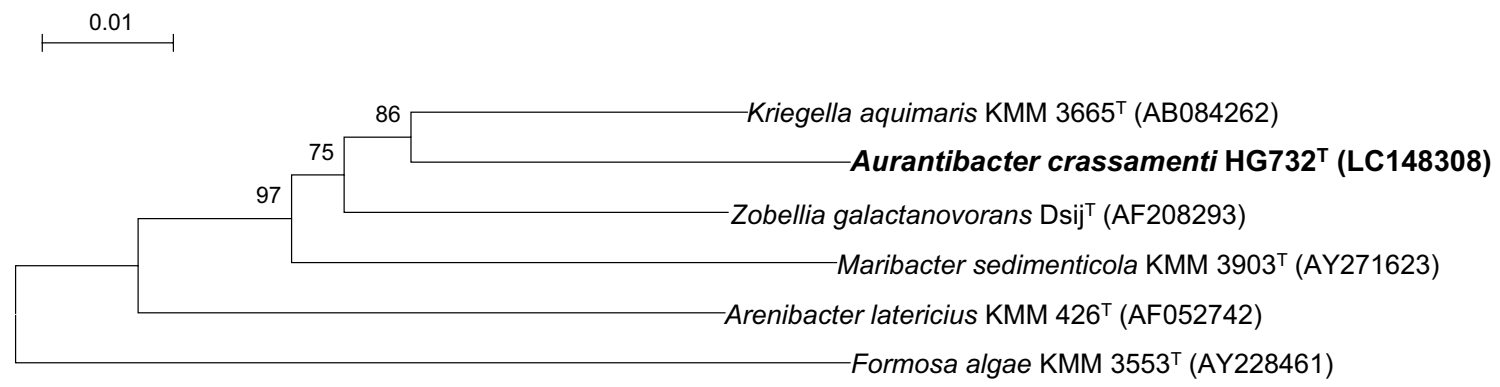

Fig. 2 Neighbor-joining tree of 16S rRNA gene sequence similarity, showing the phylogenetic position of strain $\mathrm{HG} 732^{\mathrm{T}}$ and representatives of the family Flavobacteriaceae. The sequence of Formosa algae KMM $3553^{\mathrm{T}}$ was used as an outgroup. The sequence determined in this study is shown in bold. Bootstrap values from neighborjoining analysis are shown. Bar, $1 \%$ sequence divergence 